\section{Posterior reversible encephalopathy syndrome overshadowing COVID-19: An Abstruse scenario or a conspicuous association?}

\section{Abmad J. Abdulsalam MD, Maitha A. Alkandari, MD FBPMR, Buthaina M. Alkandari, MD, FFRRCSI,} Salem A. Alkandari, MD, FBPMR

W e present a 46-year-old male who was brought to the Emergency Department with shortness of breath. The patient initially complained of a two-day history of cough, fever, and progressive dyspnea. He was obese, a heavy smoker (20 pack years), and had been a known hypertensive for the past two years; however, he denied any compliance to any of his medications. On arrival, his vital signs showed an axillary temperature of $38.9^{\circ} \mathrm{C}$, a pulse rate of 110 beats per minute, and sinus rhythm; his respiratory rate was 22 per minute, his non-invasive blood pressure was $160 / 90 \mathrm{~mm} \mathrm{Hg}$, and his oxygen saturation was $88 \%$ (on room air). A laboratory analysis revealed lymphocytopenia (800 cells $/ \mathrm{mm}^{3}$ ) with C-reactive protein elevated to 58.0 $\mathrm{mg} / \mathrm{L}$. Nasopharyngeal swab specimens were obtained from the patient, and a real-time polymerase chain reaction (RT-PCR) assay was performed, which tested positive for SARS-CoV2.

Shortly after admission, the patient's condition deteriorated to severe respiratory distress; he was therefore transferred to the Intensive Care Unit, requiring intubation and mechanical ventilation. $\mathrm{He}$ received four days of lopinavir-ritonavir and hydroxychloroquine with no clinical improvement and was subsequently put on a tracheostomy during his one-month stay in the ICU. After clinically improving, the patient was transferred to the Medical COVID Unit for supportive care and physiotherapy, specifically gait training. He remained afebrile but continued to be persistently hypertensive with a maximum systolic pressure of $195 \mathrm{~mm} \mathrm{Hg}$ despite being on $15 \mathrm{mg}$ of Lisinopril.

On day two post ICU discharge, the patient suffered an episode of confusion, blurred vision, and a tonicclonic seizure that lasted one minute and later aborted spontaneously.

A renal function test showed increased creatinine levels, indicating an acute kidney injury secondary to both the angiotensin-converting-enzyme inhibitor he was receiving and his uncontrolled blood pressure.
A computed tomography scan of the brain showed asymmetrical hypodensity within the bilateral parietooccipital white matter (Figure 1A). Brain magnetic resonance imaging revealed bilateral posterior parietooccipital, centrum semiovale, and corona radiata hyperintensities with morphological vasogenic edema consistent with non-complicated posterior reversible encephalopathy (PRES) (Figures 1B-D).

The patient was readmitted to the ICU withsupportive measurements and blood pressure control. The patient improved in that he was able to obey commands, offered no further complaints of visual impairment, and was weaned off of mechanical ventilation after 7 days. $\mathrm{He}$ remained afebrile and seizure free throughout the rest of the admission. Since then, the patient has continued on $500 \mathrm{mg}$ of Levetiracetam twice daily. Fourteen days after he was discharged from the ICU, he was transferred to the Physical Medicine and Rehabilitation Hospital in a stable condition for rehabilitation after his prolonged

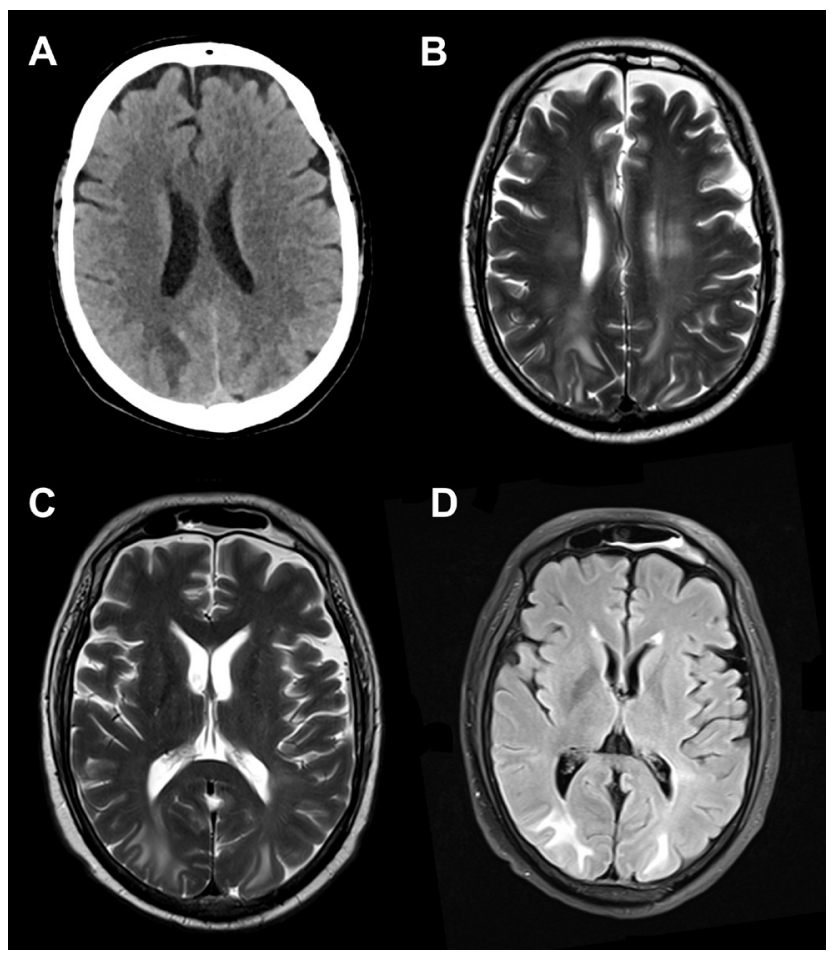

Figure 1-CT axial brain plain image (A) showed asymmetrical hypodensity within the bilateral posterior parieto-occipital white matter. B-D) T2 weight and FLAIR axial brain MRI images revealed asymmetrical bilateral posterior parieto-occipital, centrum semi ovale and corona radiata hyperintensities with morphological features of vasogenic edema consistent with non-complicated posterior reversible encephalopathy. 
stay in the ICU, remaining afebrile after 2 negative swabs for SARS-CoV2.

This case describes a patient with PRES shortly after suffering from a COVID-19 infection. The PRES is a spectrum of radiologic and clinical findings unified by acute neurological symptoms and associated cerebral vasogenic edema. ${ }^{1}$ The PRES is thought to occur as a result of disordered cerebrovascular autoregulation and is associated with acute renal failure, acute hypertension, inflammatory and autoimmune conditions, select immunomodulatory medications, and endothelial injury. ${ }^{1,2}$ Similar to our case, leading up to the development of PRES, our patient had multiple risk factors, including acute kidney injury, a history of elevated blood pressure, and an inflammatory process secondary to the infamous cytokine storm documented in COVID-19 infections. ${ }^{3,4}$ As a consequence of these morbidities, he suffered the neurological and radiological consequences of this syndrome.

Herein, similar to a handful of other cases recently published, we present similar findings concerning SARS-CoV2 infection as a potential factor in the development of PRES. ${ }^{2,4,5}$ Hence, we believe more extensive studies are warranted to clearly examine in detail the neurological relationships associated with COVID-19. In addition, we warn clinicians about possible cerebrovascular complications associated with SARS-CoV2 that may cause morbidity and mortality in patients. Last but not least, given the reversibility of this syndrome, we advocate awareness among clinicians about this rare neurological syndrome and its possible association with COVID-19. Furthermore, and perhaps more importantly, we emphasize strict blood pressure control in susceptible patients with COVID-19.

Received 18th November 2020. Accepted 8th January 2021.

From the Department of Physical Medicine and Rehabilitation (Abdulsalam, Alkandari S), Physical Medicine and Rehabilitation Hospital, Andalous, from the Department of Physical Medicine and Rehabilitation (Alkandari M), Department of Medical Imaging (Alkandari B), Jaber Al-Ahmad Hospital, Kuwait. Address correspondence and reprint requests to: Dr. Ahmad J. Abdulsalam, Department of Physical Medicine and Rehabilitation, Physical Medicine and Rehabilitation Hospital, Andalous, Kuwait. E-mail: dr.ahmad.j.abdulsalam@gmail.com

Orcid ID: https://orcid.org/0000-0002-3592-6933

\section{References}

1. Fugate JE, Rabinstein AA. Posterior reversible encephalopathy syndrome: clinical and radiological manifestations, pathophysiology, and outstanding questions. Lancet Neurol 2015; 14: 914-925.

2. Kishfy L, Casasola M, Banankhah P, Parvez A, Jan YJ, Shenoy $\mathrm{AM}$, et al. Posterior reversible encephalopathy syndrome (PRES) as a neurological association in severe Covid-19. J Neurol Sci 2020; 414: 116943.

3. Parauda SC, Gao V, Gewirtz AN, Parikh NS, Merkler AE, Lantos J, et al. Posterior reversible encephalopathy syndrome in patients with COVID-19. J Neurol Sci 2020; 416: 117019.

4. Hu B, Huang S, Yin L. The cytokine storm and COVID-19. J Med Virol 2020: doi: 10.1002/jmv.26232

5. Princiotta cariddi L, Tabaee damavandi P, Carimati F, et al. Reversible Encephalopathy Syndrome (PRES) in a COVID-19 patient. J Neurol 2020: 1-4. 